\title{
Effects of miR-340 overexpression and knockdown on the proliferation and metastasis of NSCLC cell lines
}

\author{
XIDAN ZHU, GANG TIAN, JING QUAN, PENG HE and JINBO LIU \\ Department of Laboratory Medicine, The Affiliated Hospital of Southwest Medical University, \\ Luzhou, Sichuan 646000, P.R. China
}

Received January 5, 2019; Accepted May 23, 2019

DOI: $10.3892 /$ ijmm.2019.4213

\begin{abstract}
The present study aimed to investigate the potential biological functions of microRNA-340 (miR-340) in non-small cell lung cancer (NSCLC) beyond its role as a critical regulator of tumorigenesis and tumor progression. The expression levels of miR-340 and RAB27B were analyzed by reverse transcription-quantitative polymerase chain reaction. Subsequently, the protein expression levels of RAB27A, RAB27B, RAB9A, RAB11A and BRAB21 were determined by western blot analysis. The expression levels of the aforementioned proteins in NSCLC tissues were analyzed by immunohistochemistry. RAB27B, as a potential target of miR-340 was investigated via a dual-luciferase reporter assay. The proliferative ability of PC9, A549 and BEAS-2B cells was detected with a Cell Counting kit- 8 assay, while the migration and invasion of the NSCLC cells were analyzed using a Transwell assay. The results revealed that the expression levels of miR-340 in the NSCLC cells were significantly decreased compared with those in normal cells (BEAS-2B cells). RAB27B was proposed as a potential target gene of miR-340, and its expression was notably increased in the NSCLC cells. miR-340 overexpression inhibited the migration and invasion of the NSCLC cells by targeting RAB27B, while the knockdown of miR-340 exerted opposite effects. On the whole, these findings indicate that the miR-340/RAB27B axis may be actively involved in the occurrence of NSCLC. Thus, miR-340 and RAB27B may be novel therapeutic targets for the treatment of NSCLC.
\end{abstract}

\section{Introduction}

Lung cancer accounts for the highest proportion ( 25\%) of all cancer-associated mortalities worldwide, and has a mortality rate of $>15 \%$ (1). Of all lung cancer cases, $>85 \%$ are

Correspondence to: Dr Jinbo Liu, Department of Laboratory Medicine, The Affiliated Hospital of Southwest Medical University, 25 Taiping Sreet, Luzhou, Sichuan 646000, P.R. China

E-mail: superman66996@sohu.com

Key words: microRNA-340, non-small cell lung cancer, RAB27B, migration, invasion non-small cell lung cancer (NSCLC), which is associated with a high mortality rate $(2,3)$. At present, the most effective curative method for NSCLC is surgical resection; however, surgical treatment has been successfully applied to only a few patients, as NSCLC tends to be diagnosed at advanced stages. Therefore, early diagnosis with predictive biomarkers and therapeutic applications with prognostic biomarkers are vital for improving the survival rate of patients with NSCLC.

MicroRNAs (miRNAs or miRs) are a group of non-coding RNAs (18-22 nucleotides in length) that play a role in the post-translational regulation of cell proliferation, differentiation and death, as well as other physiological and pathological processes (4-7). The role of miRNAs as tumor-inducers or -suppressors depends on their targets in numerous types of cancer. As previously reported, the dysregulation of miRNAs promotes tumor occurrence and development (8). Accumulating evidence supports the involvement of miR-340 in a variety of cancer types (9-11). miR-340 modulates the growth, migration and invasion of numerous types of cancer cells (12-15). miR-340 has been shown to suppress the migration, invasion and metastasis of breast cancer cells via the Wnt signaling pathway (16) or by downregulating Rho kinase-1 $(17,18)$; thus, miR-340 may be a key tumor suppressor in the diagnosis and treatment of breast cancer. These findings suggest that miR-340 may act as a suppressor of tumor progression. A previous study however, reported the association between miR-340 dysregulation and the development of NSCLC (19). miR-340 functions as a tumor suppressor by regulating the expression of cyclin-dependent kinase-4 in NSCLC cells, inhibiting cell growth (20). In addition, it has been demonstrated that miR-340 inhibits tumor cell proliferation and induces apoptosis by targeting a variety of negative regulators of p27 in NSCLC (21).

RABs, small $G$ proteins of the Ras superfamily, serve as regulators of vesicular transport in the exocytic and endocytic pathways in eukaryotic cells (22). It has been demonstrated that RABs play significant roles in endocytosis, cell secretion, growth and signal transduction (23). miR-30b/c has been shown to directly downregulate the expression of RAB18 and to inhibit the proliferation of NSCLC cells, indicating that $\mathrm{miR}-30 \mathrm{~b} / \mathrm{c}$ may serve as a tumor suppressor gene in the pathogenesis of NSCLC (24). An elevated expression of RAB27A has been shown to be associated with NSCLC and resistance to conventional chemotherapeutic agents (25); however, the link 
between RAB27B and miR-340 in NSCLC remains unclear. Thus, the present study aimed to investigate the function of RAB27B in association with miR-340 in NSCLC.

The findings of the aforementioned studies indicate that miR-340 may be a potential target in the treatment of NSCLC; however, the molecular mechanisms of miR-340 in modulating the expression of RAB27B in the occurrence and development of NSCLC remain unclear. Thus, the present study aimed to investigate miR-340 as a novel tumor suppressor in NSCLC.

\section{Materials and methods}

Cell culture. The human lung cancer cell lines A549, PC9 and normal bronchial epithelial cells (BEAS-2B; American Type Culture Collection) were cultured in Dulbecco's modified Eagle's medium (DMEM; Gibco; Thermo Fisher Scientific, Inc.) containing $10 \%$ fetal bovine serum (Gibco; Thermo Fisher Scientific, Inc.), $100 \mathrm{U} / \mathrm{ml}$ penicillin and $100 \mathrm{~g} / \mathrm{ml}$ streptomycin (Shanghai Xinyu Biotechnology Pharmaceutical Co. Ltd.) at $37^{\circ} \mathrm{C}$ in $5 \% \mathrm{CO}_{2}$.

Tissue collection. In total, 22 pairs of NSCLC and adjacent non-tumor tissues were obtained from patients with NSCLC who were treated at The Affiliated Hospital of Southwest Medical University between 2015 and 2018; samples were stored at $-80^{\circ} \mathrm{C}$. Written informed consent was obtained from all patients. The present study was approved by the Ethics Committee of the Affiliated Hospital of Southwest Medical University.

Immunohistochemistry (IHC). All tissues were immersed in $10 \%$ formalin and embedded in paraffin. The expression of RAB family proteins, including RAB27A, RAB27B, RAB21, RAB11A and RAB9A in the tissue samples was detected by IHC. Upon deparaffinization with xylene and rehydration with ethanol, the sections were washed with PBS twice and immersed in $\mathrm{H}_{2} \mathrm{O}_{2}$ solution to block endogenous peroxidase activity. Following washing with $\mathrm{PBS}, 10 \%$ goat serum was added for $1 \mathrm{~h}$ at room temperature to block nonspecific reactions. The tissues were then incubated overnight at $4^{\circ} \mathrm{C}$ with anti-RAB27A (dilution 1:200; cat. no. sc-74586; Santa Cruz Biotechnology, Inc.), anti-RAB27B (dilution 1:200; cat. no. DF12060; Affinity Biosciences), anti-RAB21 (dilution 1:200; cat. no. sc-81917; Santa Cruz Biotechnology, Inc.), anti-RAB11A (dilution 1:200; cat. no. sc-166912; Santa Cruz Biotechnology, Inc.) and anti-RAB9A (dilution 1:200; cat. no. sc-71950; Santa Cruz Biotechnology, Inc.) antibodies. Subsequently, the sections were washed with PBS twice and incubated with a horseradish peroxidase (HRP)-conjugated secondary antibody (dilution 1:200; cat. no. sc-2347; Santa Cruz Biotechnology, Inc.) at $37^{\circ} \mathrm{C}$ for $30 \mathrm{~min}$. Finally, the sections were incubated with 3,3'-diaminobenzidine to detect proteins. The samples were visualized under a light microscope (Nikon Corp.).

Cell transfection. The BEAS-2B, A549 and PC9 cells were seeded into 6 -well plates at a density of $5 \times 10^{4}$ cells/well and transfected with miR-340 mimics, miR-340 inhibitors or mock miRNA (Invitrogen; Thermo Fisher Scientific, Inc.) using Lipofectamine ${ }^{\circledR} 2000$ (Invitrogen; Thermo Fisher Scientific, Inc.) according to the manufacturer's protocols. Total protein was isolated from the transfected cells with protein extraction kit (KeyGen Biotech) according to the manufacturer's instructions at $4^{\circ} \mathrm{C}$, then total protein was quantified using a BCA assay (KeyGen Biotech) according to the manufacturer's instructions at $4^{\circ} \mathrm{C}$. Total RNA was extracted from the transfected cells using TRIzol reagent (Invitrogen; Thermo Fisher Scientific, Inc.) and quantified using NanoDrop ${ }^{\circledR}$ One/One C Micro-UV-Vis Spectrophotometer (Thermo Fisher Scientific, Inc.). Total protein and RNA was used for further analysis. The sequences of miR-340 mimics and miR-340 inhibitors were as follows: miR-340 mimics, UUAUAAAGCAAUGAG ACUGAUU and miR-340 inhibitors, AAUCAGUCUCAU UGAUUUAUAA.

Cell Counting kit-8 (CCK-8) assay. The proliferation of the transfected BEAS-2B, A549 and PC9 cells was assessed using a CCK-8 assay (Sigma-Aldrich; Merck KGaA). Briefly, the cells were seeded into 24 -well plates and cultured for $24 \mathrm{~h}$, and then transfected with miR-340 mimics, miR-340 inhibitors or mock miRNA. Subsequently, $100 \mu 1$ transfected cells were added to 96 -well plates at a density of $2 \times 10^{4}$ cells/well, and $10 \mu \mathrm{l} \mathrm{CCK}-8$ solution were added to each well, followed by $2 \mathrm{~h}$ of incubation at $37^{\circ} \mathrm{C}$. Viable cells were counted with BioTek Epoch microplate absorbance reader (Bio-Tek Instruments, Inc.) at $450 \mathrm{~nm}$.

Luciferase reporter assays. Potential targets of miR-340 were predicted using TargetScan (www.targetscan.org/vert_71/) and further confirmed by MicroRNA.org30 (www.microrna. org), and miRBase (http://www.mirbase.org/). To further confirm whether RAB27B is a direct target of miR-340, luciferase reporter experiments were performed. The cells plated into 24-well plates at $40 \%$ confluence were co-transfected for $24 \mathrm{~h}$ with miR-340 mimics, miR-340 inhibitors or mock miRNA and luciferase reporter plasmids [pGL3 RAB27B 3'-untranslated region (UTR) wild-type or mutant; Genepharm, Inc.] using Lipofectamine 2000 according to the manufacturer's instructions. Luciferase activity was measured 24 h later with the Dual-Luciferase ${ }^{\circledR}$ Assay (Promega Corp.). Lightswitch Renilla activity was normalized to pGL3 to assess transfection efficiency. Each assay was conducted in triplicate.

Reverse transcription-quantitative polymerase chain reaction $(R T-q P C R)$. Total RNA was isolated from NSCLC tissues, non-tumor tissues and cell lines using TRIzol reagent for RT-qPCR analysis. miRNA or mRNA expression was evaluated by two-step RT-qPCR. cDNA was prepared using A-MLV reverse transcriptase (Invitrogen; Thermo Fisher Scientific, Inc.); qPCR with SYBR-Green dye was performed according to the manufacturer's protocols (Takara Inc.). For qPCR, cDNA $(0.5 \mu \mathrm{l})$, forward primer $(0.5 \mu \mathrm{l})$, reverse primer $(0.5 \mu \mathrm{l})$, deoxyribonucleotide triphosphate mixture $(2 \mu 1,2.5 \mathrm{mM})$, DNA polymerase $(0.5 \mu \mathrm{l}, 10 \mathrm{U} / \mu \mathrm{l}), 5 \mathrm{X}$ buffer $(6 \mu \mathrm{l})$ and double distilled $\mathrm{H}_{2} \mathrm{O}(15 \mu \mathrm{l})$ were subjected to 32 cycles at $94^{\circ} \mathrm{C}$ for $5 \mathrm{~min}, 95^{\circ} \mathrm{C}$ for $15 \mathrm{sec}$ and $56^{\circ} \mathrm{C}$ for $35 \mathrm{sec}$. Each sample was evaluated in duplicate, and the mean quantification cycle $(\mathrm{Cq})$ was calculated. 
The following forward and reverse primers were used: 5'-GCGCTAGTTTCCTGT-3' and 5'-GTGCAGGGTCCG AGGT-3' (miR-340); 5'-TGCGGGACAAGAGCGGTTCCG-3' and 5'-GCCAGTTCCCGAGCTTGCCGT T-3' (RAB27B); and 5'-CAATGACCCCTTCATTGACC-3' and 5'-GACAAGCTT CCCGTTCTCAG-3' (GAPDH). PCR amplification of miRNA was conducted using miRNA-specific forward primers and the universal poly(T) adaptor reverse primer with U6 as an internal control. The forward sequence of Homo sapiens miR-340 was 5'-GCTTATAAAGCAATGAGACTGATT-3'. U6 was used as an internal control (forward, 5'-CTCGCTTCG GCAGCACA-3' and reverse, 5'-AACGCTTCACGAATTTG CGT-3'). The results were quantified using the $2^{-\Delta \Delta C q}$ method as previously described (26).

Transwell assay. The effects of miR-340 on A549 cell invasion and migration were analyzed using Transwell assays (Costar, Aiyan Biotechnology Co. Ltd.) with or without Matrigel (Clontech, Laboratories, Inc.), respectively. The A549 cells transfected for $24 \mathrm{~h}$ were resuspended in the upper 24-well chambers at a density of $1 \times 10^{5}$ cells/well. Serum-free DMEM and 10\% FBS-DMEM were added to the upper and lower chambers, respectively. After $24 \mathrm{~h}$, cells on the upper membrane were collected using cotton swabs, while cells in the lower chamber were fixed with $100 \%$ methanol for $15 \mathrm{~min}$ and stained with $0.05 \%$ crystal violet (Beyotime Biotechnology Company) for $20 \mathrm{~min}$ at $37^{\circ} \mathrm{C}$. Invasive and migrated cells were observed under a inverted Fluorescence Microscope IX53 (Olympus). Each assay was conducted in triplicate.

Western blot analysis. Total proteins were extracted from tissues and transfected cells using lysis buffer, and the protein concentration was determined using a BCA protein assay kit. Proteins $(20 \mu \mathrm{g})$ were subjected to $10 \%$ SDS-PAGE and transferred onto polyvinylidene difluoride membranes. The membranes were blocked with $2 \%$ bovine serum albumin and cultured with primary antibodies against RAB27A (dilution 1:600; cat. no. sc-74586; Santa Cruz Biotechnology, Inc.), RAB27B (dilution 1:800; cat. no. DF12060; Affinity Biosciences), RAB21 (dilution 1:1,000; cat. no. sc-81917; Santa Cruz Biotechnology, Inc.), RAB11A (dilution 1:1,200; cat.no. sc-166912; Santa Cruz Biotechnology, Inc.) and RAB9A (dilution 1:1,000; cat. no. sc-71950; Santa Cruz Biotechnology, Inc.) for $60 \mathrm{~min}$ at $37^{\circ} \mathrm{C}$. Following 3 washes with TBST, the membranes were incubated with goat anti-rabbit IgG-HRP secondary antibody (dilution 1:1,000; cat. no. sc-2004; Santa Cruz Biotechnology, Inc.) at room temperature for $30 \mathrm{~min}$ and then washed as aforementioned. Subsequently, specific binding was detected with the chemiluminescence (GE Healthcare Life Sciences). The detection of the chemiluminescent signal was performed in the gel documentation system ImageQuant LAS 4000 Mini (GE Healthcare Life Sciences). The intensity of the bands corresponding to the target proteins was analyzed using Image J 1.8.0 (National Institutes of Health).

Statistical analysis. Data were analyzed with SPSS 19.0 (IBM Corp., Armonk, NY, USA) and expressed as the means \pm standard error of the mean. Differences between groups were compared by one-way analysis of variance followed by a Tukey's post-hoc test. $\mathrm{P}<0.05$ was considered to indicate a statistically significant difference. Each experiment was performed at least 3 times.

\section{Results}

miR-340 expression is downregulated in NSCLC tissues and cell lines. The present study analyzed the expression levels of miR-340 and RAB27B in 22 pairs of NSCLC tissues, adjacent tissues and nornal BEAS-2B cells or NSCLC cell lines (A549 and PC9) by RT-qPCR; the protein expression of RAB27B in the NSCLC cell lines was determined by western blot analysis. The results revealed that miR-340 expression was significantly decreased in NSCLC tissues (Fig. 1A) and cell lines (Fig. 1B). On the contrary, the mRNA expression levels of RAB27B were significantly increased in NSCLC tissues (Fig. 1C) and cell lines (Fig. 1D), while RAB27B protein expression was markedly upregulated in the NSCLC cell lines (Fig. 1E and F). Thus, the downregulation of miR-340 may be associated with the upregulation of RAB27B in NSCLC.

Analysis of the RAB family of proteins in NSCLC tissues. In order to examine the expression of RAB family proteins in NSCLC tissues, the expression levels of 5 RAB proteins, including RAB27A, RAB27B, RAB21, RAB11A and RAB9A, were evaluated by immunohistochemical analysis in NSCLC and adjacent tissues (Fig. 2). The results indicated that the protein expression levels of RAB27A, RAB27B, RAB21, RAB11A and RAB9A were notably increased in NSCLC tissues compared with non-tumor tissues (Fig. 2).

miR-340 targets $R A B 27 B$ and directly decreases its expression. The present study revealed that RAB27B may be a target of miR-340 using predictive tools, including TargetScan, miRanda and miRBase. Of note, a putative binding site of miR-340 was identified in the 3'-UTR of RAB27B (Fig. 3A). The luciferase activity of the RAB27B wild-type 3'-UTR reporter was markedly suppressed by transfection with miR-340 mimics compared with the negative control, while the mutant luciferase reporter was significantly activated by transfection with miR-340 inhibitors (Fig. 3B). miR-340 expression was significantly increased in all cell lines transfected with miR-340 mimics, whereas it was decreased in NSCLC cells transfected with miR-340 inhibitors (Fig. 3C). RAB27B mRNA expression was notably decreased following transfection with miR-340 mimics; however, RAB27B expression in the A549 cells was upregulated in response to the silencing of miR-340 with miR-340 inhibitors (Fig. 3D). These results indicate that miR-340 regulates the post-transcriptional expression of RAB27B by directly binding to its 3'-UTR.

Effects of miR-340 overexpression on the proliferation of NSCLC cell lines. The effects of miR-340 overexpression or knockdown on the proliferation of the BEAS-2B, A549 and PC9 cells were evaluated by a CKK-8 assay (Fig. 4). It was observed that transfection with miR-340 mimics significantly suppressed the proliferative ability of these cell lines; however, the cell proliferative ability was markedly promoted following the knockdown of miR-340 with inhibitors. 
A

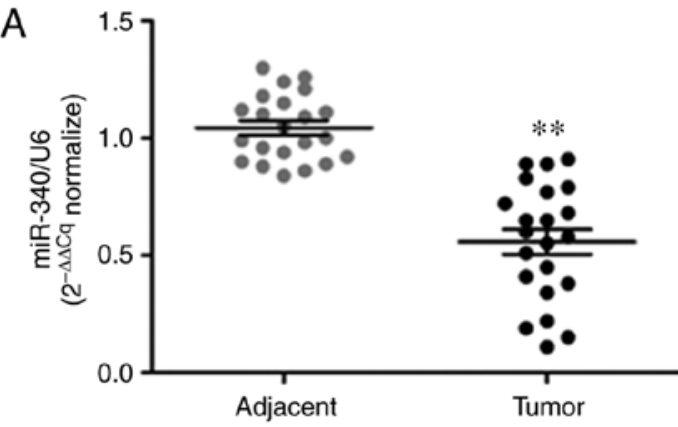

C

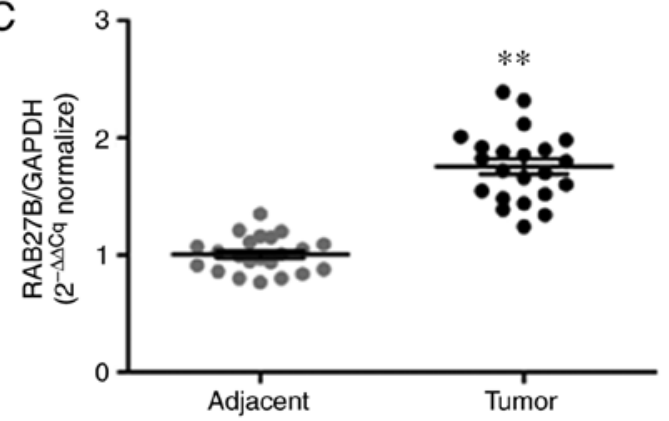

E

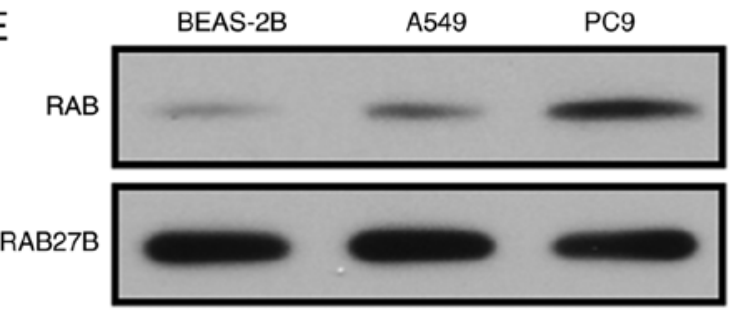

B
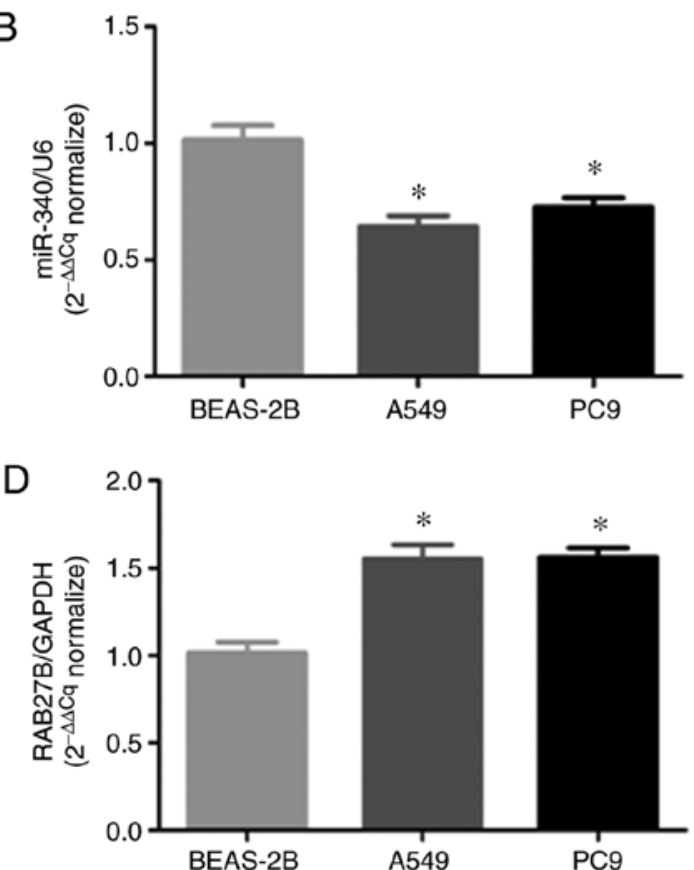

$\mathrm{F}$

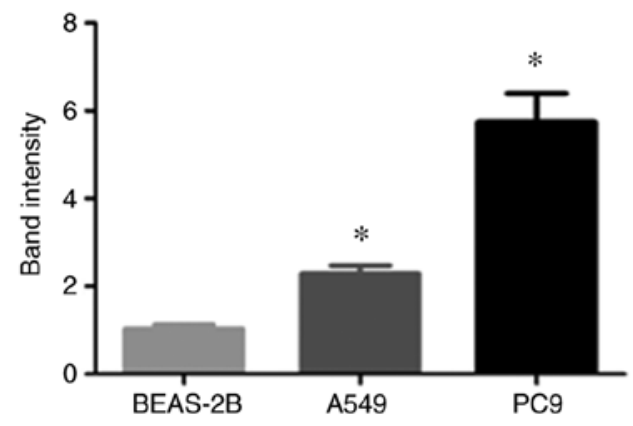

Figure 1. Expression levels of miR-340 and RAB27B in NSCLC tissues and cell lines. (A) RT-qPCR analysis of miR-340 expression levels in 22 paired human NSCLC and adjacent tissues. (B) Quantitative analysis of miR-340 expression in NSCLC cell lines. (C) RT-qPCR analysis of RAB27B mRNA expression in 22 paired NSCLC tissues. (D) Quantitative analysis of RAB27B gene expression in NSCLC cell lines. (E) RAB27B protein expression in NSCLC cell lines, as determined by western blotting. (F) The expression levels of RAB27B protein were significantly increased in NSCLC cell lines A549 and PC9 compared with BEAS-2B cells. All experiments were performed in triplicate and the data are presented as the means \pm standard error of the mean. ${ }^{*} \mathrm{P}<0.05$ vs. normal cells (BEAS-2B); and ${ }^{* * *} \mathrm{P}<0.01$ vs. adjacent tissues. RT-qPCR, reverse transcription-quantitative polymerase chain reaction; miR, microRNA; NSCLC, non-small cell lung cancer.
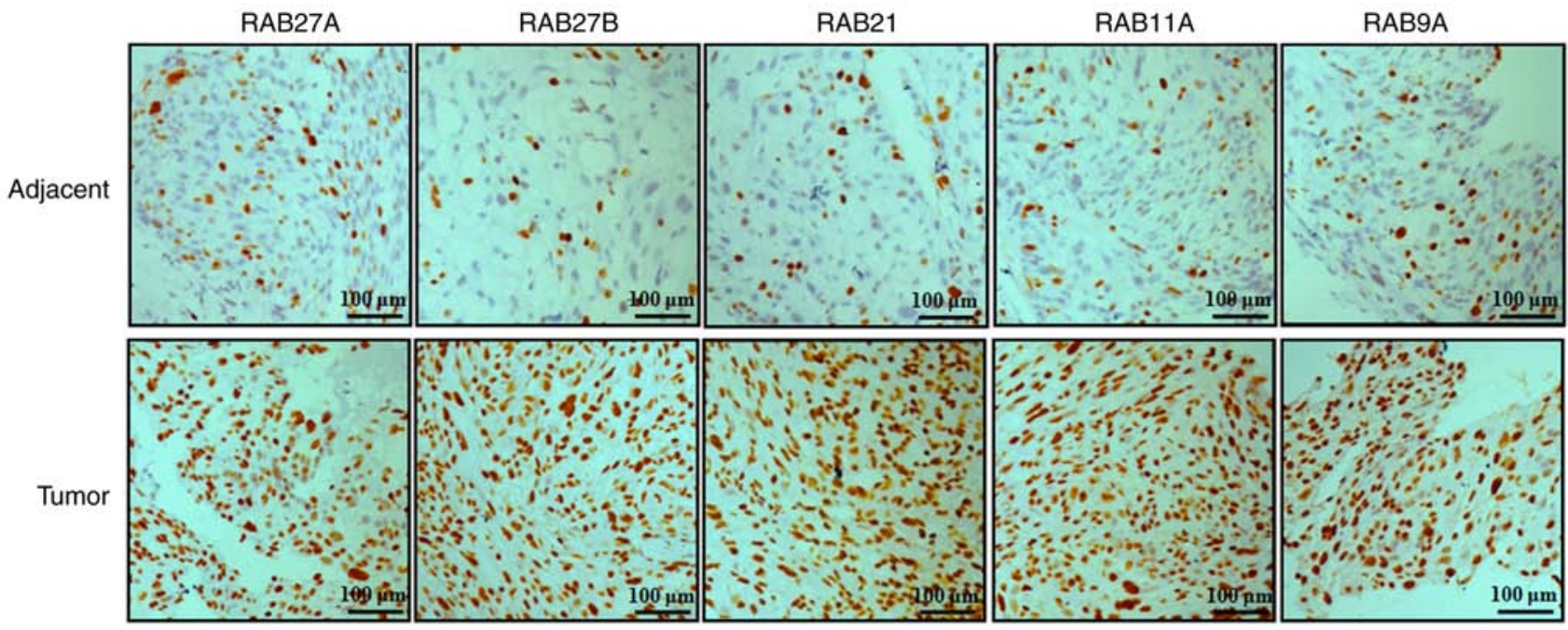

Figure 2. Immunohistochemical analysis of RAB family proteins in NSCLC tissues. The expression of the RAB family of proteins, including RAB27A, $\mathrm{RAB} 27 \mathrm{~B}, \mathrm{RAB} 21, \mathrm{RAB} 11 \mathrm{~A}$ and RAB9A in tumor and adjacent tissues of patients with NSCLC was analyzed by immunohistochemistry and observed under an inverted microscope. NSCLC, non-small cell lung cancer. 

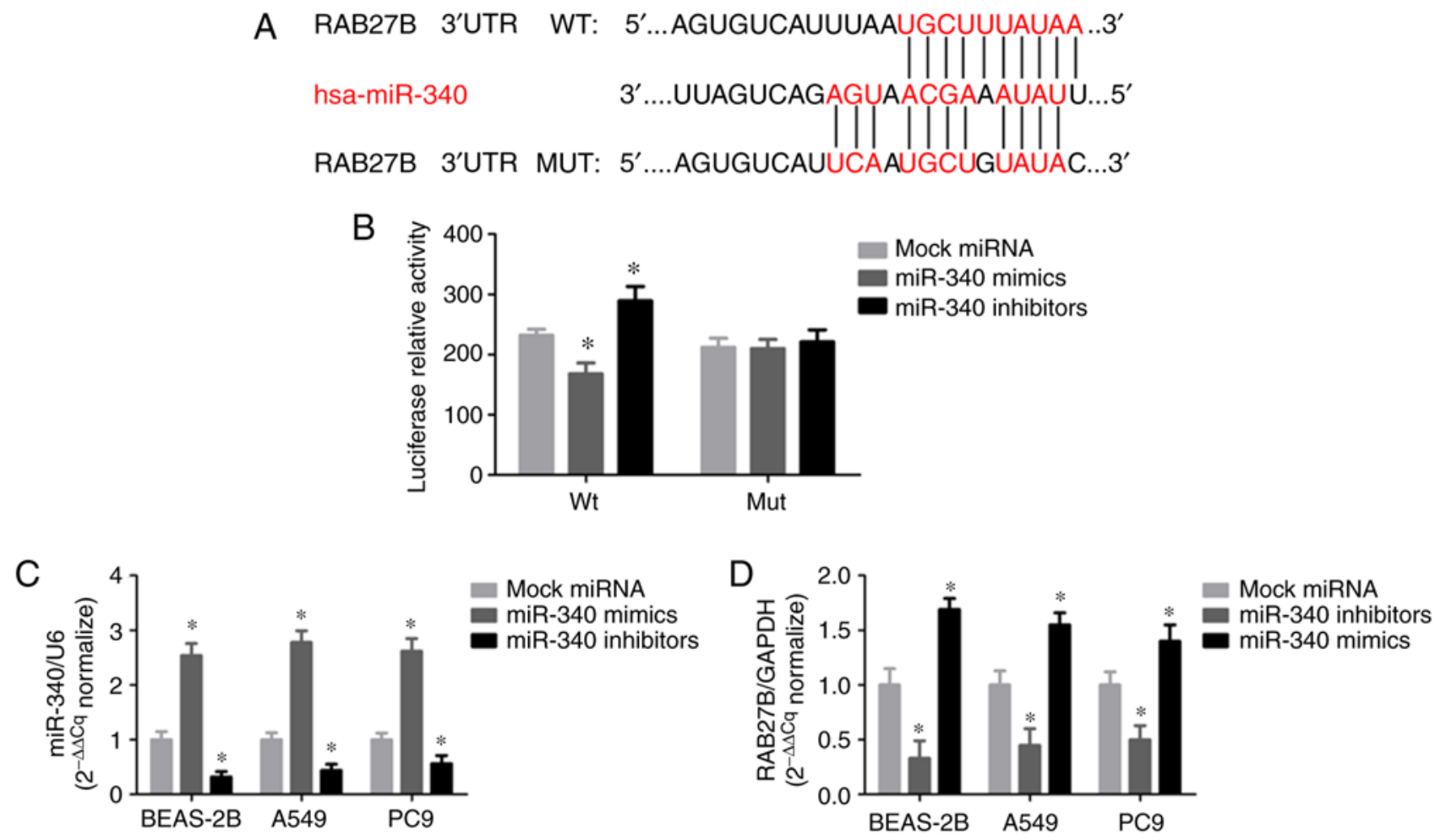

Figure 3. miR-340 targets RAB27B and directly decreases its expression. (A) Putative miR-340-binding sequence in the 3'-UTR of RAB27B mRNA. Mutations were generated in the RAB27B 3'-UTR sequence, specifically in the sites complementary to miR-340. (B) The wild-type or mutant reporter constructs were co-transfected with miR-340 mimics into A549 cells and analyzed by a luciferase reporter assay. (C) Quantitative analysis of miR-340 expression in A549 cells transfected with miR-340 mimics or miR-340 inhibitors. (D) Reverse transcription-quantitative polymerase chain reaction analysis of RAB27B mRNA expression in A549 cells transfected with miR-340 mimics or miR-340 inhibitors. All experiments were performed at least 3 times and data are presented as the means \pm standard error of the mean. ${ }^{*} \mathrm{P}<0.05$ vs. normal cells (BEAS-2B). miR, microRNA; UTR, untranslated region.
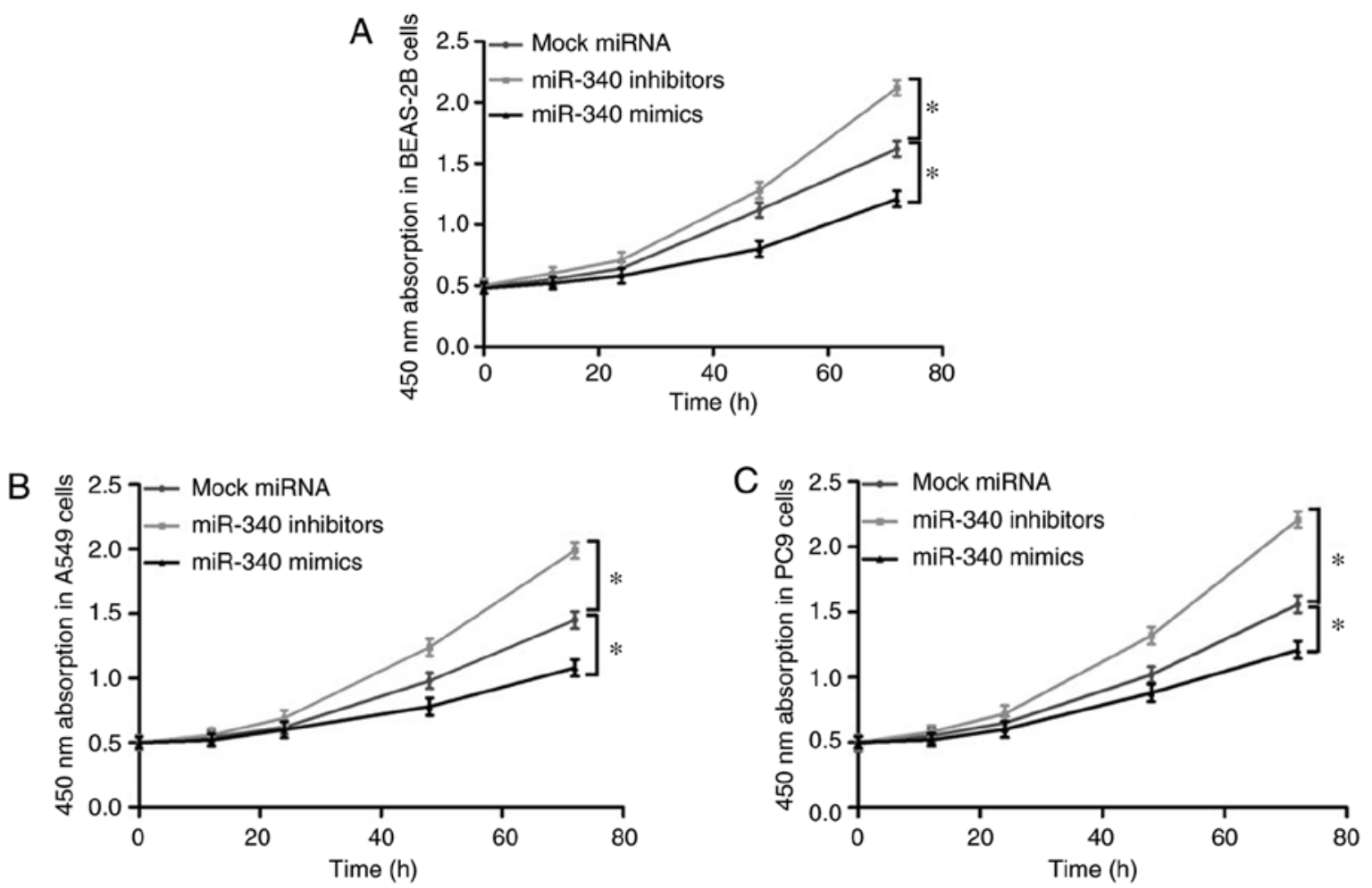

Figure 4. Overexpression of miR-340 suppresses the proliferation of non-small cell lung cancer cells in vitro, as determined by a Cell Counting kit-8 assay. (A) Proliferation of BEAS-2B cells transfected with miR-340 mimics or inhibitors compared with the mock miRNA group. (B) Proliferation of A549 cells transfected with miR-340 mimics or inhibitors compared with mock miRNA-transfected cells. (C) Viability of PC9 cells transfected with miR-340 mimics or miR-340 inhibitors compared with those transfected with mock miRNA. The absorbance values at $450 \mathrm{~nm}$ are presented as the means \pm standard error of the mean. ${ }^{*} \mathrm{P}<0.05$. miR/miRNA, microRNA. 
A

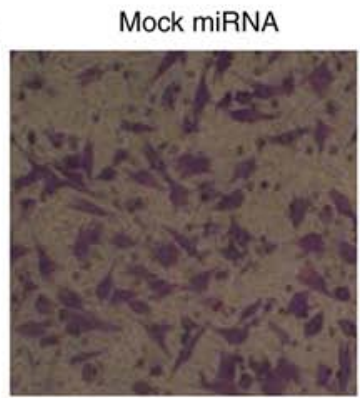

C

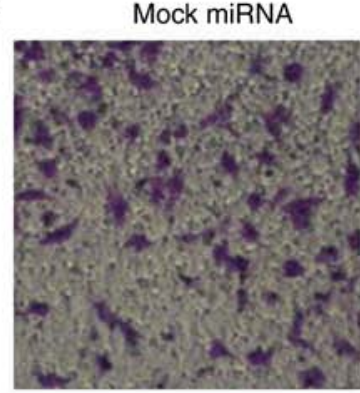

miR-340 inhibitors

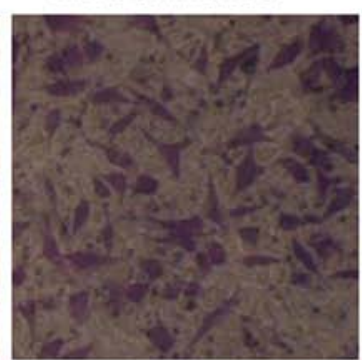

miR-340 inhibitors

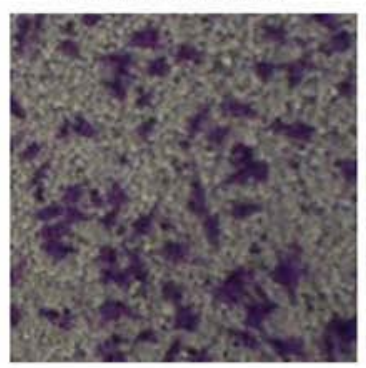

miR-340 mimics

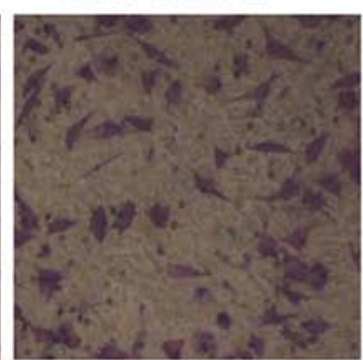

miR-340 mimics

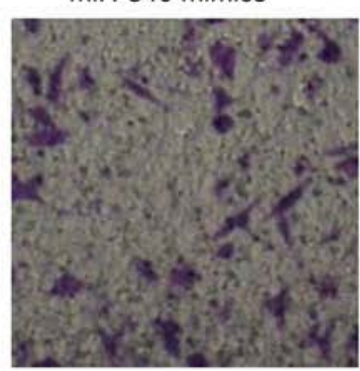

B

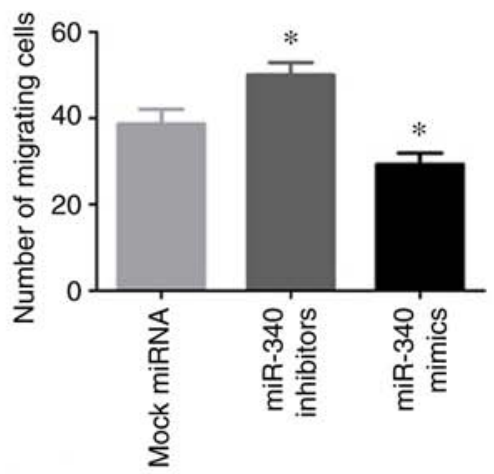

D

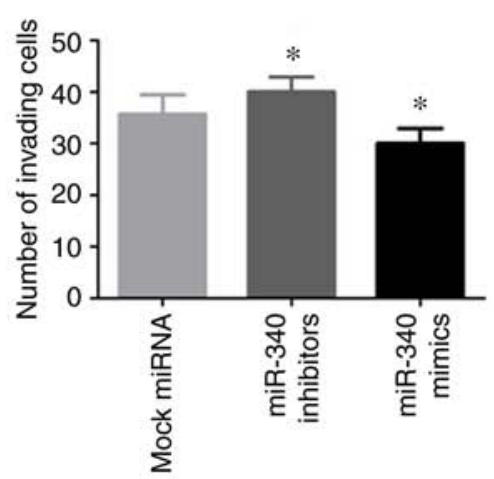

Figure 5. Overexpression of miR-340 suppresses the migration and invasion of non-small cell lung cancer cells. (A and B) The migration of A549 cells transfected with miR-340 mimics, miR-340 inhibitors or mock miRNA was analyzed by a Transwell assay. Knockdown of miR-340 promoted the migration of A549 cells, while the overexpression of miR-340 suppressed migration. (C and D) Invasion of A549 cells transfected with miR-340 mimics, miR-340 inhibitors or mock miRNA, as detected by a Transwell assay. The invasive ability of A549 cells was promoted by knockdown of miR-340, while miR-340 overexpression exhibited opposing effects. All experiments were performed in triplicate and data are presented as the means \pm standard error of the mean. ${ }^{*} \mathrm{P}<0.05$ vs. mock miRNA. $\mathrm{miR} / \mathrm{miRNA}$, microRNA.

Effects of miR-340 on the migratory and invasive abilities of NSCLC cells. To enhance our understanding of the biological functions of miR-340 in NSCLC cells, the A549 cells were transfected with miR-340 mimics or inhibitors, and the migratory and invasive abilities of the cells were investigated by a Transwell assay. The results revealed that transfection with miR-340 mimics notably inhibited A549 cell migration, while transfection with miR-340 inhibitors exerted opposite effects (Fig. 5A and B). Furthermore, transfection with miR-340 mimics suppressed cell invasion, and this was promoted by transfection with miR-340 inhibitors (Fig. 5C and D).

In vitro analysis of the effects of miR-340 on RAB27 proteins. The protein expression levels of RAB27A and RAB27B were markedly decreased in the miR-340 mimic-transfected A549 cells, but were increased upon transfection with miR-340 inhibitors (Fig. 6A and B). In addition, transfection with miR-340 mimics significantly suppressed the expression levels of RAB9A, RAB11A and RAB21 in A549 cells, while miR-340 inhibitors exerted opposite effects (Fig. 6C and D).

\section{Discussion}

It is well known that one miRNA can target numerous genes. The function of miR-340 in the occurrence of various types of cancer has been widely studied in recent years. As previously reported, miR-340 restricts the development of breast cancer cells by targeting numerous oncogenes, including c-Met, zinc finger E-box-binding homeobox 1 and enhancer of zeste homolog 2 (27-29). Furthermore, miR-340 has been shown to inhibit the progression of glioma by targeting cyclin-dependent kinase 6 , cyclin-D1/D2 and tissue plasminogen activator $(30,31)$. In addition, miR-340 has been shown to play a suppressive role in lung, colorectal (32), ovarian (33) and prostate cancers (34), laryngeal squamous cell carcinoma (35) and osteosarcoma (36); however, the role of miR-340 in NSCLC remains unknown. The present study reported that miR-340 was downregulated in NSCLC, whereas miR-340 overexpression suppressed the growth and migration of NSCLC cells. This suggests that miR-340 may play a tumor-suppressing role; however, a previous study revealed that miR-340 played an oncogenic role in gastric cancer by targeting cyclin G2 (37). The biological function of miR-340 may depend on its target gene, which is mainly modulated by miR-340.

Various studies have demonstrated that the RAB family of proteins play important roles in NSCLC. The downregulation of RAB27A has been shown to suppress the proliferative, migratory and invasive abilities of NSCLC cells in vitro, and to inhibit the growth of xenograft tumors in mice, indicating that RAB27A may be a potential therapeutic target in NSCLC (38). miR-451 regulates the survival of NSCLC cells via the downregulation of RAB14, suggesting that targeting the interaction between miR-451 and RAB14 may be a novel therapeutic target in NSCLC (39). miR-30b/c directly targets and downregulates the expression of RAB18 to inhibit the proliferation of NSCLC cells. RAB11A protein has been shown to be overexpressed in NSCLC tissues, and to be associated with advanced tumor, node and metastasis stage, positive nodal status and poor 

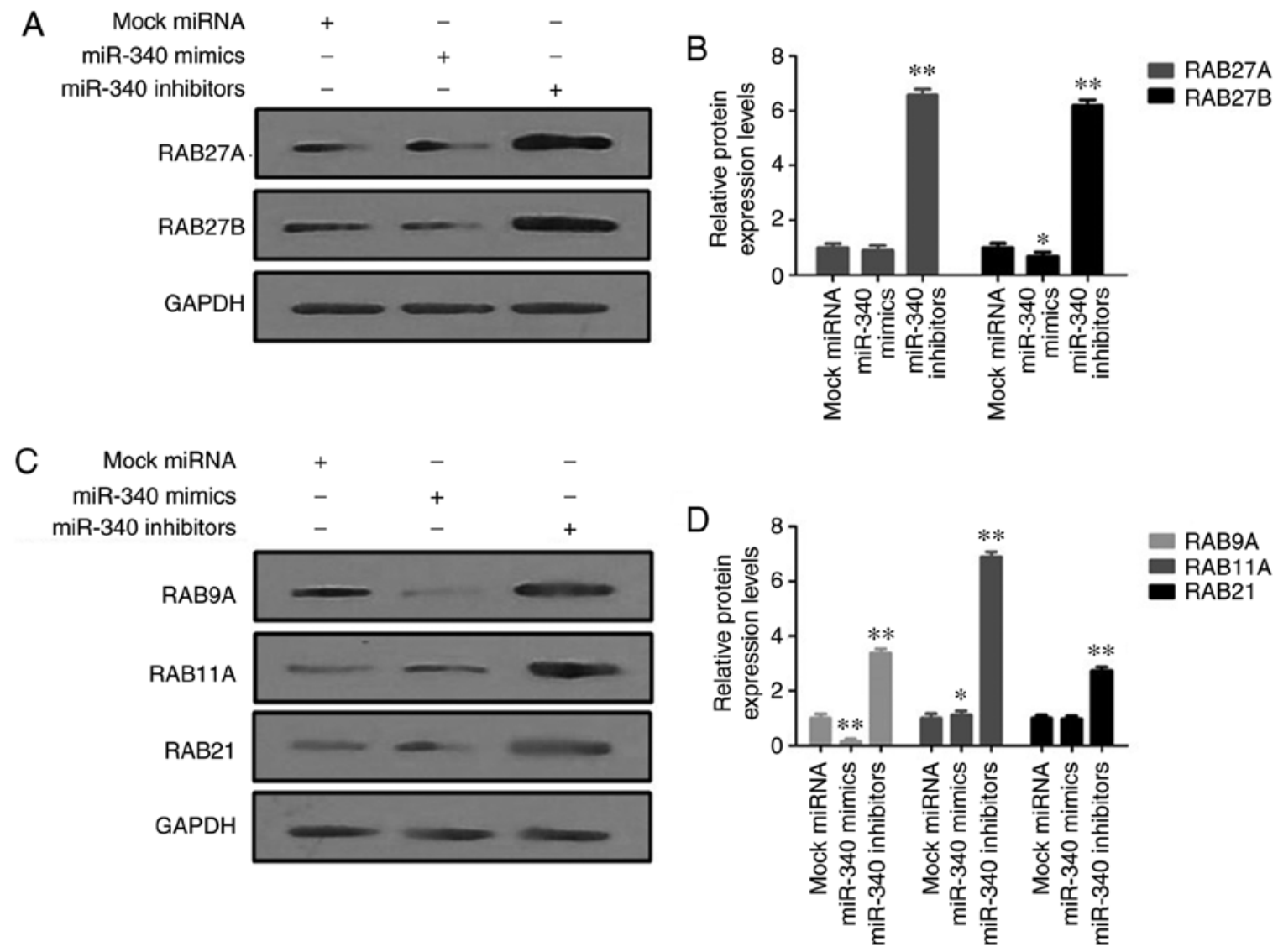

Figure 6. Expression levels of RAB27B and other proteins of the RAB family. (A and B) RAB27B and RAB27A protein expression levels in A549 cells transfected with miR-340 mimics or inhibitors were examined by western blot analysis. (C and D) Western blot analysis was applied to analyze the expression levels of RAB9, RAB11A and RAB21 in A549 cells transfected with miR-340 mimics or inhibitors. Data are presented as the means \pm standard error of the mean of 3 independent experiments. ${ }^{*} \mathrm{P}<0.05$, and ${ }^{* *} \mathrm{P}<0.01$ vs. mock miRNA. miR, microRNA.

patient prognosis. In addition, the overexpression of RAB11A has been shown to promote the proliferation, invasion and migration of NSCLC cells via the upregulation of cyclin D1 and cyclin E, and the downregulation of p27 (40).

RAB27, a member of the small GTPase family (41), has been reported to be associated with various human cancers. For instance, RAB27A has been identified as an inducer of melanoma growth (42), while the inhibition of RAB27A in melanoma cell lines has been shown to suppress primary tumor growth and lung metastasis (43). The nuclear factor- $\kappa$ B-mediated RAB27A expression facilitates cytokine secretion to promote the stemness of colon cancer cells (44). RAB27A expression has been associated with tumor grade and the unfavorable prognosis of glioma (45). These studies indicate RAB27A as a potential tumor-promoting protein in human cancers, whereas others have suggested that RAB27A serves as a tumor suppressor. RAB27A upregulation has been shown to be associated with the favorable prognosis of patients with colorectal cancer (46). Furthermore, RAB27B facilitates the invasion and migration of estrogen receptor-positive breast cancer cells (47); the downregulated expression RAB27A and $\mathrm{RAB} 27 \mathrm{~B}$ has been detected in the late stages of prostate cancer (48).

Few studies have reported the association between RAB and miR-340 in NSCLC; thus, TargetScan software was employed in the present study, which predicted RAB11A,
RAB27B and RAB43 as potential targets of miR-340. RAB27B was proposed to be the most likely target of miR-340 of the aforementioned RAB proteins. In addition, we also conducted a luciferase reporter experiment to confirm whether RAB27B is a direct target of miR-340. The present study identified RAB27B as a novel target gene of miR-340, and observed that miR-340 was downregulated, while RAB27B was upregulated in NSCLC tissues and cell lines. miR-340 overexpression inhibited the proliferation and invasion of NSCLC cells; these effects were reversed by the knockdown of RAB27B. The mechanisms underlying the effects of miR-340 comprise the targeting and inhibition of RAB27B by miR-340. Furthermore, the miR-340/RAB27B axis may be involved in the occurrence and progression of NSCLC.

Previous reports have demonstrated that RAB27A and RAB27B are the major components involved in vesicle fusion and trafficking, and exosome secretion, playing important roles in tumor progression and metastasis. The increased expression of RAB27B has been observed in hepatocellular carcinoma (49), colorectal cancer (50) and breast cancer (51), indicating that RAB27B may be a valuable predictor of metastasis and prognosis, or a potential therapeutic target for the treatment of various types of cancer. The upregulated expression of RAB27B may be an unfavorable prognostic factor in patients with squamous cell carcinoma of the lungs (52). 
Additionally, the upregulated expression of RAB27B has been shown to be associated with the malignant features of lung adenocarcinoma (LUAD); RAB27B was identified as a potential indicator of metastasis and the prognosis of LUAD (53).

In conclusion, miR-340 plays a critical role in NSCLC, and its overexpression restricts the growth and invasion of NSCLC cells by downregulating RAB27B. The results of the present study may provide novel insight into the molecular mechanisms underlying the occurrence and development of NSCLC, However, the potential regulatory mechanisms of miR-340 targeting RAB27B require further investigation.

\section{Acknowledgements}

Not applicable.

\section{Funding}

This study was supported by grants from the large data system platform for laboratory medicine consultation oriented to precision medicine (2017TJPT0003).

\section{Availability of data and materials}

All data generated or analyzed during this study are included in this published article or are available from the corresponding author on reasonable request.

\section{Authors' contributions}

$\mathrm{XZ}$ and JL conceived and designed the experiments. XZ, GT, JQ and PH performed these experiments and analyzed the data. XZ and JL drafted and revised the manuscript. All authors have read and approved the final manuscript.

\section{Ethics approval and consent to participate}

The present study was approved by the Ethics Committee of the Affiliated Hospital of Southwest Medical University. Written informed consent was obtained from all patients.

\section{Patient consent for publication}

Not applicable.

\section{Competing interests}

All the authors declare that there are no any competing interests.

\section{References}

1. Siegel R, Ma J, Zou Z and Jemal A: Cancer statistics, 2014. CA Cancer J Clin 64: 9-29, 2014

2. Ferlay J, Soerjomataram I, Dikshit R, Eser S, Mathers C, Rebelo M, Parkin DM, Forman D and Bray F: Cancer incidence and mortality worldwide: Sources, methods and major patterns in GLOBOCAN 2012. Int J Cancer 136: E359-E386, 2015.

3. Rossi A, Maione P, Sacco PC, Sgambato A, Casaluce F, Ferrara ML, Palazzolo G, Ciardiello F and Gridelli C: ALK inhibitors and advanced non-small cell lung cancer (Review). Int J Oncol 45: 499-508, 2014.
4. Raitoharju E, Seppala I, Oksala N, Lyytikainen LP, Raitakari O, Viikari J, Ala-Korpela M, Soininen P, Kangas AJ, Waldenberger M, et al: Blood microRNA profile associates with the levels of serum lipids and metabolites associated with glucose metabolism and insulin resistance and pinpoints pathways underlying metabolic syndrome: The cardiovascular risk in young finns study. Mol Cell Endocrinol 391: 41-49, 2014.

5. Jiang $\mathrm{F}, \mathrm{Yu} \mathrm{Q}$, Chu Y, Zhu X, Lu W, Liu Q and Wang Q: MicroRNA-98-5p inhibits proliferation and metastasis in non-small cell lung cancer by targeting TGFBR1. Int J Oncol 54: 128-138, 2019.

6. Tang Q, Li M, Chen L, Bi F and Xia H: miR-200b/c targets the expression of RhoE and inhibits the proliferation and invasion of non-small cell lung cancer cells. Int J Oncol 53: 1732-1742, 2018.

7. Lujambio A and Lowe SW: The microcosmos of cancer. Nature 482: 347-355, 2012.

8. Othman $\mathrm{N}$ and Nagoor NH: Overexpression of miR-361-5p plays an oncogenic role in human lung adenocarcinoma through the regulation of SMAD2. Int J Oncol 54: 306-314, 2019.

9. Rongxin S, Pengfei L, Li S, Xiaochen J and Yihe H: MicroRNA-340-5p suppresses osteosarcoma development by down-regulating the $\mathrm{Wnt} / \beta$-catenin signaling pathway via targeting the STAT3 gene. Eur Rev Med Pharmacol Sci 23: 982-991, 2019.

10. Shi Z, Li Y, Qian X, Hu Y, Liu J, Zhang S and Zhang J: miR-340 inhibits triple-negative breast cancer progression by reversing EZH2 mediated miRNAs dysregulated expressions. J Cancer 8: 3037-3048, 2017.

11. Wei P, Qiao B, Li Q, Han X, Zhang H, Huo Q and Sun J: microRNA-340 suppresses tumorigenic potential of prostate cancercells by targeting high-mobility group nucleosome-binding domain 5. DNA Cell Biol 35: 33-43, 2016.

12. Huang D, Qiu S, Ge R, He L, Li M, Li Y and Peng Y: miR-340 suppresses glioblastoma multiforme. Oncotarget 6: 9257-9270, 2015.

13. Huang T, Zhou Y, Zhang J, Wong CC, Li W, Kwan JSH, Yang R, Chan AKY, Dong Y, Wu F, et al: SRGAP1, a crucial target of miR-340 and miR-124, functions as a potential oncogene in gastric tumorigenesis. Oncogene 37: 1159-1174, 2018.

14. Xie L, Chen Z, Liu H, Guan L, Wang Z and Li W: Effects of miR-340 on hepatocellular carcinoma by targeting the DcR3 gene. Dig Liver Dis 50: 291-296, 2018.

15. Zhao P, Ma W, Hu Z, Zhang Y, Zhang S and Wang Y: Up-regulation of miR-340-5p promotes progression of thyroid cancer by inhibiting BMP4. J Endocrinol Invest 41: 1165-1172, 2018.

16. Chen CP, Sun ZL, Lu X, Wu WX, Guo WL, Lu JJ, Han C, Huang JQ and Fang Y: miR-340 suppresses cell migration and invasion by targeting MYO10 in breast cancer. Oncol Rep 35: 709-716, 2016.

17. Mohammadi-Yeganeh S, Paryan M, Arefian E, Vasei M, Ghanbarian H, Mahdian R, Karimipoor M and Soleimani M: MicroRNA-340 inhibits the migration, invasion, and metastasis of breast cancer cells by targeting Wnt pathway. Tumour Biol 37: 8993-9000, 2016.

18. Maskey N, Li D, Xu H, Song H, Wu C, Hua K, Song J and Fang L: MicroRNA-340 inhibits invasion and metastasis by down-regulating ROCK1 in breast cancer cells. Oncol Lett 14: 2261-2267, 2017.

19. Zhang Z, Wang Y, Zhang W, Li J, Liu W and Lu W: Long non-coding RNA SNHG14 exerts oncogenic functions in non-small cell lung cancer through acting as a miR-340 sponge. Biosci Rep 39: pii: BSR20180941, 2019.

20. Qin Y, Zhou X, Huang C, Li L, Liu H, Liang N, Chen Y, Ma D, Han Z, Xu X, et al: Lower miR-340 expression predicts poor prognosis of non-small cell lung cancer and promotes cell proliferation by targeting CDK4. Gene 675: 278-284, 2018.

21. Fernandez S, Risolino M, Mandia N, Talotta F, Soini Y, Incoronato M, Condorelli G, Banfi S and Verde P: miR-340 inhibits tumor cell proliferation and induces apoptosis by targeting multiple negative regulators of p27 in non-small cell lung cancer. Oncogene 34: 3240-3250, 2015.

22. Chia WJ and Tang BL: Emerging roles for Rab family GTPases in human cancer. Biochim Biophys Acta 1795: 110-116, 2009.

23. Bobrie A, Krumeich S, Reyal F, Recchi C, Moita LF, Seabra MC, Ostrowski M and Théry C: Rab27a supports exosome-dependent and -independent mechanisms that modify the tumour microenvironment and can promote tumour progression. Cancer Res 72: 4920-4930, 2012. 
24. Zhong K, Chen K, Han L and Li B: MicroRNA-30b/c inhibits non-small cell lung cancer cell proliferation by targeting Rab18. BMC Cancer 14: 703, 2014

25. Li W, Mu D, Tian F, Hu Y, Jiang T, Han Y, Chen J, Han G and Li X: Exosomes derived from Rab27a-overexpressing tumor cells elicit efficient induction of antitumor immunity. Mol Med Rep 8: 1876-1882, 2013.

26. Livak KJ and Schmittgen TD: Analysis of relative gene expression data using real-time quantitative PCR and the 2(-Delta Delta C(T)) method. Methods 25: 402-408, 2001

27. Rezaei Z, Sebzari A, Kordi-Tamandani DM and Dastjerdi K: Involvement of the dysregulation of miR-23b-3p, miR-195-5p, miR-656-5p, and miR-340-5p in trastuzumab resistance of HER2-positive breast cancer cells and system biology approach to predict their targets involved in resistance. DNA Cell Biol 38: 184-192, 2019.

28. Wu ZS, Wu Q, Wang CQ, Wang XN, Huang J, Zhao JJ, Mao SS, Zhang GH, Xu XC and Zhang N: miR-340 inhibition of breast cancer cell migration and invasion through targeting of oncoprotein c-Met. Cancer 117: 2842-2852, 2011.

29. Hou LK, Yu Y, Xie YG, Wang J, Mao JF, Zhang B, Wang X and Cao XC: miR-340 and ZEB1 negative feedback loop regulates TGF- $\beta$-mediated breast cancer progression. Oncotarget 7 : 26016-26026, 2016

30. Li X, Gong X, Chen J, Zhang J, Sun J and Guo M: miR-340 inhibits glioblastoma cell proliferation by suppressing CDK6, cyclin-D1 and cyclin-D2. Biochem Biophys Res Commun 460: 670-677, 2015

31. Yamashita D, Kondo T, Ohue S, Takahashi H, Ishikawa M Matoba R, Suehiro S, Kohno S, Harada H, Tanaka J and Ohnishi T: miR340 suppresses the stem-like cell function of glioma-initiating cells by targeting tissue plasminogen activator. Cancer Res 75: 1123-1133, 2015.

32. Takeyama $\mathrm{H}$, Yamamoto $\mathrm{H}$, Yamashita $\mathrm{S}, \mathrm{Wu} X$, Takahashi $\mathrm{H}$, Nishimura J, Haraguchi N, Miyake Y, Suzuki R, Murata K, et al: Decreased miR-340 expression in bone marrow is associated with liver metastasis of colorectal cancer. Mol Cancer Ther 13: 976-985, 2014

33. Li P, Sun Y and Liu Q: MicroRNA-340 induces apoptosis and inhibits metastasis of ovarian cancer cells by inactivation of NF-x03BA;B1. Cell Physiol Biochem 38: 1915-1927, 2016.

34. Huang K, Tang Y, He L and Dai Y: MicroRNA-340 inhibits prostate cancer cell proliferation and metastasis by targeting the MDM2-p53 pathway. Oncol Rep 35: 887-895, 2016.

35. Yu W, Zhang G, Lu B, Li J, Wu Z, Ma H, Wang H and Lian R: miR-340 impedes the progression of laryngeal squamous cell carcinoma by targeting EZH2. Gene 577: 193-201, 2016.

36. Zhou X, Wei M and Wang W: MicroRNA-340 suppresses osteosarcoma tumor growth and metastasis by directly targeting ROCK1. Biochem Biophys Res Commun 437: 653-658, 2013.

37. Yin G, Zhou H, Xue Y, Yao B and Zhao W: MicroRNA-340 promotes the tumor growth of human gastric cancer by inhibiting cyclin G2. Oncol Rep 36: 1111-1118, 2016.

38. Li X, Wang H, Ni Q, Tang Z, Ni J, Xu L, Huang H, Ni S and Feng J: Effects of silencing Rab27a gene on biological characteristics and chemosensitivity of non-small cell lung cancer. Oncotarget 8: 94481-94492, 2017.

39. Wang R, Wang ZX, Yang JS, Pan X, De W and Chen LB: MicroRNA-451 functions as a tumor suppressor in human non-small cell lung cancer by targeting ras-related protein 14 (RAB14). Oncogene 30: 2644-2658, 2011.

40. Dong Q, Fu L, Zhao Y, Du Y, Li Q, Qiu X and Wang E: Rablla promotes proliferation and invasion through regulation of YAP in non-small cell lung cancer. Oncotarget 8: 27800-27811, 2017.
41. Imai A, Yoshie S, Ishibashi K, Haga-Tsujimura M, Nashida T, Shimomura $\mathrm{H}$ and Fukuda M: EPI64 pr parotid acinar cells otein functions as a physiological GTPase-activating protein for RAB27 protein and regulates amylase release in rat. J Biol Chem 286: 33854-33862, 2011.

42. Akavia UD, Litvin O, Kim J, Sanchez-Garcia F, Kotliar D, Causton HC, Pochanard P, Mozes E, Garraway LA and Pe'er D An integrated approach to uncover drivers of cancer. Cell 143: $1005-1017,2010$

43. Peinado H, Alečković M, Lavotshkin S, Matei I, Costa-Silva B, Moreno-Bueno G, Hergueta-Redondo M, Williams C, García-Santos G, Ghajar C, et al: Melanoma exosomes educate bone marrow progenitor cells toward a pro-metastatic phenotype through MET. Nat Med 18: 883-891, 2012.

44. Feng F, Jiang Y, Lu H, Lu X, Wang S, Wang L, Wei M, Lu W, $\mathrm{Du} Z$, Ye Z, et al: Rab27A mediated by NF- $\kappa \mathrm{B}$ promotes the stemness of colon cancer cells via up-regulation of cytokine secretion. Oncotarget 7: 63342-63351, 2016

45. Wang H, Zhao Y, Zhang C, Li M, Jiang C and Li Y: Rab27a was identified as a prognostic biomaker by mRNA profiling, correlated with malignant progression and subtype preference in gliomas. PLoS One 9: e89782, 2014.

46. Shi C, Yang X, Ni Y, Hou N, Xu L, Zhan F, Zhu H, Xiong L and Chen P: High RAB27A expression indicates favorable prognosis in CRC. Diagn Pathol 10: 68, 2015.

47. Hendrix A, Maynard D, Pauwels P, Braems G, Denys H, Van den Broecke R, Lambert J, Van Belle S, Cocquyt V, Gespach C, et al: Effect of the secretory small GTPase RAB27B on breast cancer growth, invasion, and metastasis. J Natl Cancer Inst 102: 866-880, 2010

48. Worst TS, Meyer Y, Gottschalt M, Weis CA, von Hardenberg J, Frank C, Steidler A, Michel MS and Erben P: RAB27A, RAB27B and VPS36 are downregulated in advanced prostate cancer and show functional relevance in prostate cancer cells. Int J Oncol 50: 920-932, 2017.

49. Yang X, Ye X, Sun L, Gao F, Li Y, Ji X, Wang X, Feng Y and Wang X: Downregulation of serum RAB27B confers improved prognosis and is associated with hepatocellular carcinoma progression through PI3K-AKT-P21 signaling. Oncotarget 8: 61118-61132, 2017

50. Bao J, Ni Y, Qin H, Xu L, Ge Z, Zhan F, Zhu H, Zhao J, Zhou X, Tang $X$ and Tang L: Rab27b is a potential predictor for metastasis and prognosis in colorectal cancer. Gastroenterol Res Pract 2014: 913106, 2014

51. Hendrix A, Braems G, Bracke M, Seabra M, Gahl W, De Wever O and Westbroek W: The secretory small GTPase Rab27B as a marker for breast cancer progression. Oncotarget 1: 304-308, 2010.

52. Koh HM and Song DH: Prognostic role of Rab27A and Rab27B expression in patients with non-small cell lung carcinoma. Thorac Cancer 10: 143-149, 2019.

53. Zhang L, Fan W, Xu L, Mao Q, Chen Y, Mao Y, Xu L and Wang J: Rab27b is a potential indicator for lymph node metastasis and unfavorable prognosis in lung adenocarcinoma. Dis Markers 2018: 7293962, 2018.

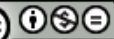

This work is licensed under a Creative Commons Attribution-NonCommercial-NoDerivatives 4.0 International (CC BY-NC-ND 4.0) License. 